\title{
Effects of ultrasound on the corneal endothelium: II. The endothelial repair process
}

\author{
LANCE E. OLSON, JOHN MARSHALL, NOEL S. C. RICE, AND \\ ROBERT ANDREWS
}

From the Departments of Clinical Ophthalmology and Visual Science, Institute of Ophthalmology, London

SUMMARY The responses of corneal endothelium to a 2-minute exposure of ultrasound from a clinical phacoemulsification device have been studied by specular microscopy and both scanning and transmission electron microscope. Healing of lesions occurred in two ways, and these were related to lesion size. Small lesions healed by a process of rosette formation, and large lesions were repaired by a process of endothelial dedifferentiation and migration. All damaged areas were re-covered with endothelial cells within 24 hours of exposure, and at this time corneal thickness had returned to control levels. The implications are discussed in relation to the phacoemulsification procedure.

The physiological processes which maintain the transparency of the cornea are ultimately dependent on the ability of the corneal endothelium to pump fluids out of the corneal stroma and into the aqueous (Hodson and Miller, 1976). Any agent which damages this system reduces the efficiency of the pumping mechanism and results in corneal swelling or oedema.

Endothelial damage has been reported following the completion of phacoemulsification for cataract removal (Polack and Sugar, 1976; Binder et al., 1976) and recently in response to the isolated ultrasonic radiation component of this procedure (Olson et al., 1978). These reports found that the degree of endothelial damage was related to the duration of the operative procedure and suggest that corneal oedema should be a common postoperative complication of this technique. Reported results indicate that transient induced oedema decreases with increasing experience, with incidence as high as $18 \%$ during early use of the instrument (Hiles and Hurite, 1973) and zero when the surgeon is proficient (Kelman, 1974). The occurrence of persistent oedema is far less common, with figures varying between zero (Kelman, 1974) and 2\% (Cleasby et al., 1974).

The apparent lack of correlation between the morphological damage seen in the corneal endothelium and the clinical observations on corneal oedema suggests a rapid healing process in response to this type of injury.

Address for reprints: Mr Noel S. C. Rice, FRCS, Moorfields Eye Hospital, City Road, London ECIV 2PD.
The present paper investigates the behaviour of the corneal endothelium during the first week after exposure to ultrasonic radiation.

\section{Materials and methods}

IN VIVO EXPERIMENTS

Adult Dutch rabbits weighing between 2 and $3 \mathrm{~kg}$ were anaesthetised with intravenous pentobarbitone before being given a retrobulbar injection of $0.5 \mathrm{ml}$ of $1 \%$ lignocaine. With the aid of an operating microscope a 3-mm incision was made at the limbus and the tip of a Cavitron-Kelman phacoemulsifier (Model 7007) was introduced into the anterior chamber parallel to the iris and at a depth approaching the edge of the pupil. Care was taken to avoid contact with endothelium and the lens capsule. All corneas were exposed to 2 minutes of ultrasound, with the generator fully tuned and a power setting of 8. Glutathione-bicarbonate-Ringer (GBR) solution was used for irrigation thoughout these experiments. After exposure, the tip was withdrawn and the limbal wound was sutured with 8-0 silk. In all animals wound closure was followed by an injection of $0.5 \mathrm{ml}$ of Gentamicin (20000 IU) into the subconjunctival space. The contralateral eye of each animal served as a control and received identical treatment except that ultrasound was withheld. No steroids were given during the postoperative period. The animals were allowed to recover for various times after exposure (1 hour, 2 hours, 8 hours, 24 hours, 48 hours, and 1 week) before being killed with intravenous pentobarbitone. 
The eyes were immediately removed and mounted in a specular microscope (Maurice, 1968), where measurements were made of corneal thickness, and the unfixed endothelium was photographed. Eyes were then fixed and processed for both scanning electron microscopy (SEM) and transmission electron microscopy (TEM) as previously described (Olson et al., 1978).

\section{IN VITRO EXPERIMENTS}

In this series of experiments animals of similar age and weight were killed with intravenous pentobarbitone and their eyes were immediately enucleated. The corneal thicknesses were measured with a specular microscope, and then the eyes were transported at room temperature in a dewar to a second laboratory. The minimum journey time was 45 minutes. The surgical procedures for both experimental and control eyes were identical to those described for in vivo experiments, except that in these eyes the anterior chamber which remained formed throughout closure was manometrically adjusted to $15 \mathrm{mmHg}$. The eyes were then returned to the initial laboratory and again mounted in specular microscopes but this time fitted with constant temperature devices maintained at $37^{\circ} \mathrm{C}$. Photographs of the endothelia and measurements of corneal thickness were made at 30-minute intervals for periods of up to 5 hours. Several of these specimens were removed at various time periods and fixed and processed for examination by both SEM and TEM.

\section{Results}

\section{SPECULAR MICROSCOPY}

The specular microscope enabled direct observation of the dynamic processes involved in endothelial repair. Two repair mechanisms were observed, and they were clearly related to the size of the area of damage. In small lesions, those involving the loss of 4 to 6 cells, the deficient area was rapidly covered by a process involving the unaffected adjacent cells (Fig. 1). Within an hour these cells had begun extending sheet-like pseudopodia along Descemet's membrane into the damaged region. These rosette formations (Oh and Evans, 1960) took between 1 and 6 hours completely to re-cover the denuded region. At this time the component cells of the rosettes were radially arranged and shaped like elongated deltoids. Once organised, most of these formations remained stable during the period they were observed under the specular microscope. However, in some rosettes one of the component cells would migrate into the centre of the array: When such an event occurred, the other cells reorganised to become almost indistinguishable from the normal endothelial mosaic.

Large lesions were difficult to study under the specular microscope because of the limited field of view. Detailed studies were therefore carried out with the scanning electron microscope.

SCANNING ELECTRON MICROSCOPY

The above observations on small lesions were confirmed by SEM and several further findings became apparent by this technique. The cells participating in the rosettes showed a marked loss of microvilli and other surface structures, and their cell borders became less well defined. Each cell became thinned and wedge-shaped, inclining towards the centre of the rosettes (Fig. 2a). The sheet-like pseudopodia were seen to end in multiple conventional or tubular pseudopodia. As these processes extended along Descemet's membrane to re-cover the denuded area, debris was pushed along in front of them until the rosette was completed (Fig. 2b). Many rosettes were therefore seen with clusters of debris located at the centre of the radial array (Fig. 3a). In most cases such debris consisted of organelles or isolated nuclei from ruptured cells.

In large lesions the responses of cells adjacent to the damaged area were slower, and migrational activity was not apparent until at least 2 hours after the ultrasonic exposure. At this time cells adjacent to the damaged area dissociated themselves from their neighbours and assumed fibroblast-like forms. This dedifferentiation and migration process extended to include cells some 3 to 6 rows away from the edge of the original lesion (Fig. $3 b$ ). With increasing time these cells became larger in surface area, but correspondingly they became extremely thin with enlarged and prominent nuclei (Fig. 4a). As they migrated into the area of the lesion the shapes of the cells varied, with both a spindle form and an amoeboid form with long fine pseudopodia being equally numerous. When individual cells reached the centre of the area of exposed Descemet's membrane they began to reform intercellular associations, thus covering the area with a 'pig-skin graft'-like process (Fig. $4 b$ ). This process continued until the entire area was re-covered, in most cases within $\mathbf{2 4}$ hours. At this time, as in the small lesions, any residual debris tended to be located at the lesion centre. By 48 hours the cells had begun to lose their fibroblastic appearance and were reorganising into a more conventional endothelial mosaic. In most lesions after 48 hours debris had dispersed, and the area of the lesion could only be determined by the presence of abnormally large or irregularly-shaped endothelial cells. At 1 week after exposure no 
difference could be determined between control and experimental tissues.

TRANSMISSION ELECTRON MICROSCOPY In the small lesions the pseudopodia-like projection of adjacent cells invaded the damaged area almost immediately after the ultrasonic exposure (Olson et al., 1978). By 1 hour these processes were some way into the damaged areas, moving over the surface of Descemet's membrane and beneath any
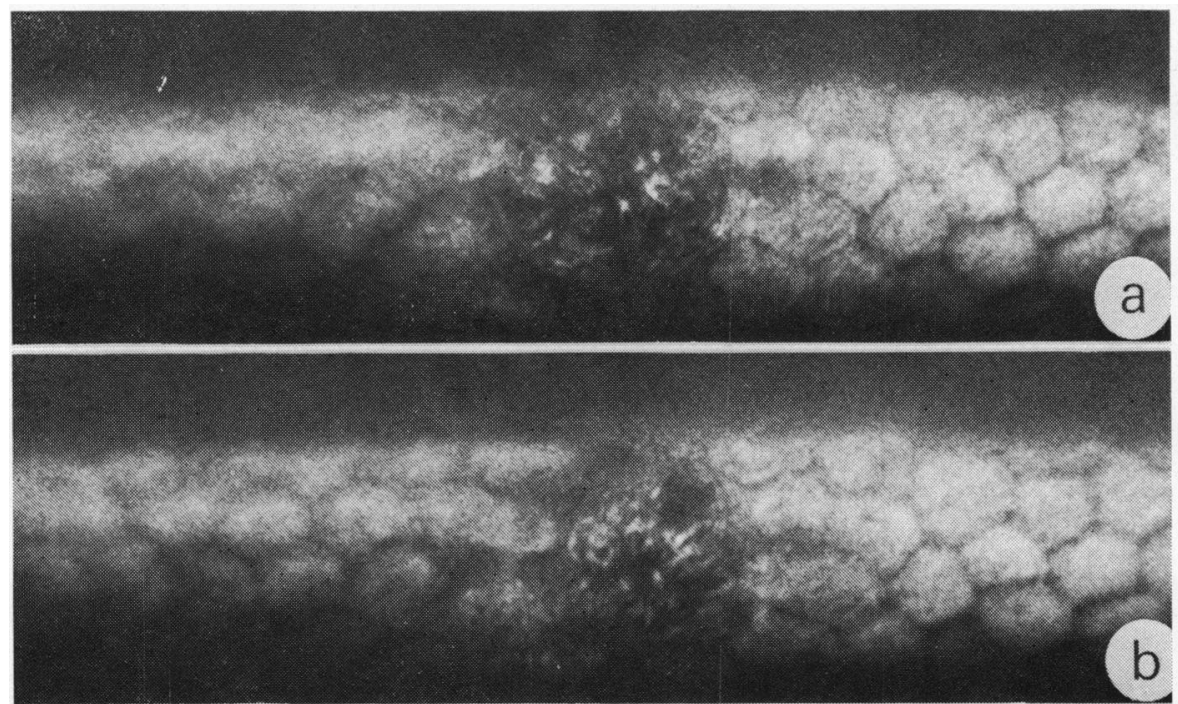

Fig. 1 Specular

photomicrographs of rabbit corneal endothelium in vitro at various times after a 2-minute exposure to ultrasound. (a) Immediately after exposure showing the loss of 5 to 6 endothelial cells, (b) 30 minutes later, (c) after 1 hour, (d) 2 hours, and (e) $2 \frac{1}{2}$ hours. The rosette formation becomes apparent after 1 hour and complete recoverage of the damaged area is achieved by $2 \frac{1}{2}$ hours. Bar marker $=40 \mu \mathrm{m}$ 

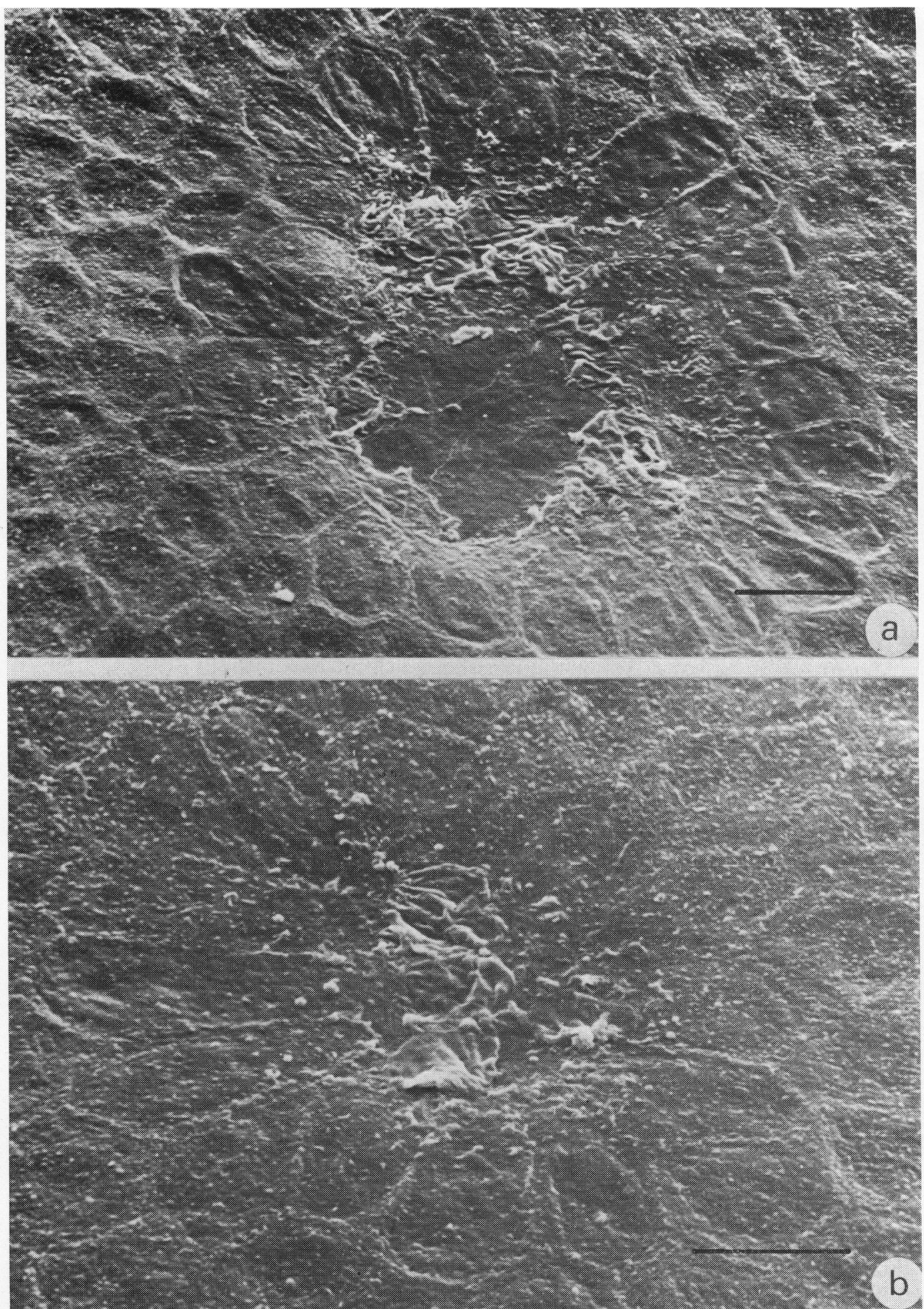

Fig. 2 Scanning electron micrographs of endothelial rosette formation: (a) 1 hour and (b) 2 hours after ultrasonic insult. The pseudopodia-like processes seen in (a) extend across the denuded area of Descemet's membrane until contact is made with similar processes from other adjacent cells (b). Bar markers $=20 \mu \mathrm{m}$ 


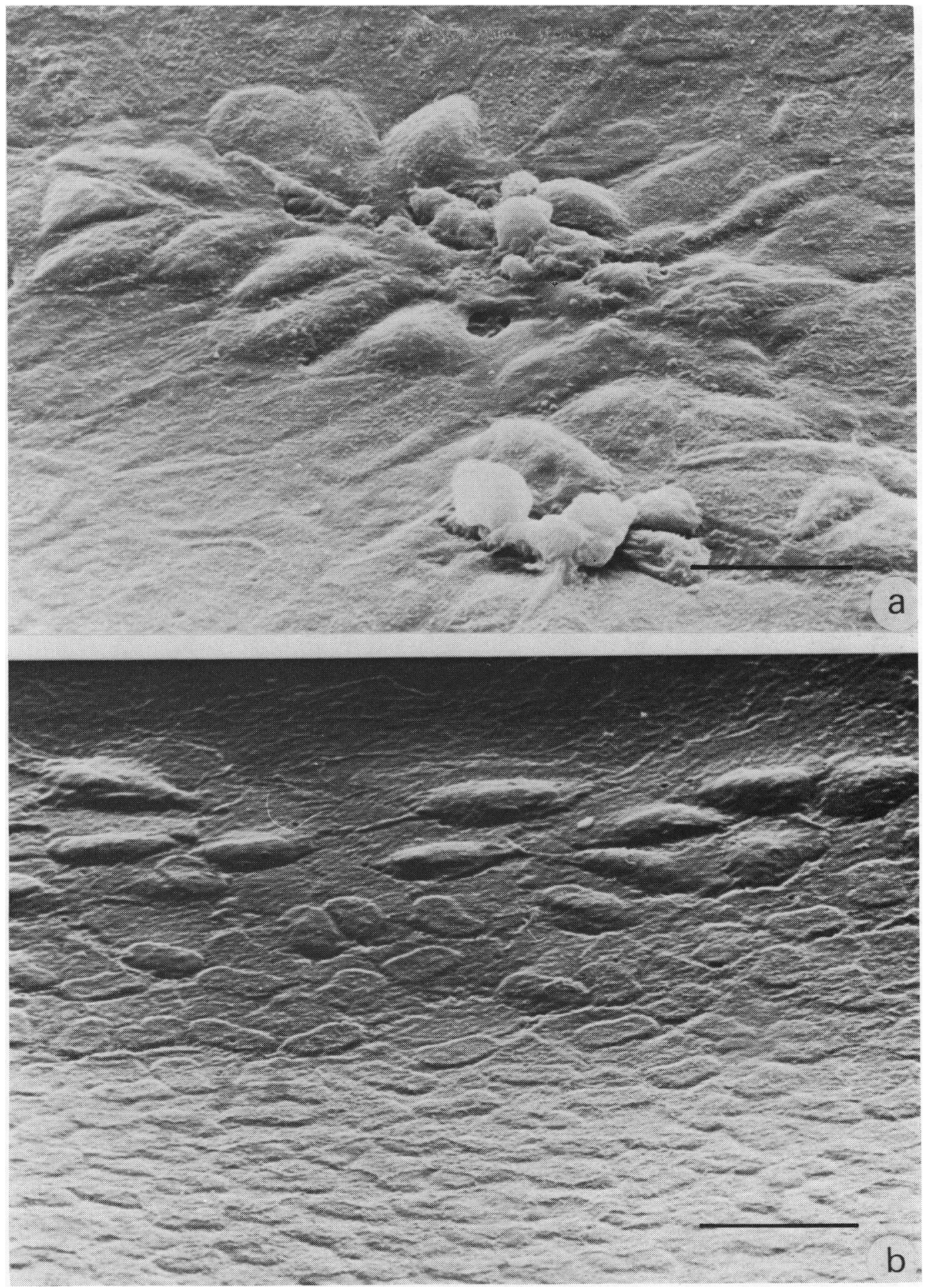

Fig. 3 (a) Scanning electron micrograph of endothelial rosettes 4 hours after ultrasonic insult. Debris can be seen on the surface of the endothelial cells and is located over the centre of the rosette. The debris is usually remnants of ruptured endothelial cells, and it arrives at the lesion centre because it is pushed by the invading pseudopodia of adjacent cells. (b) Scanning electron micrograph of dedifferentiating and migrating endothelial cells at the edge of a large lesion. This process may extend to involve cells up to 6 rows away from the initial edge of the damaged area. Bar markers: $a=20 \mu \mathrm{m}, \mathrm{b}=40 \mu \mathrm{m}$ 

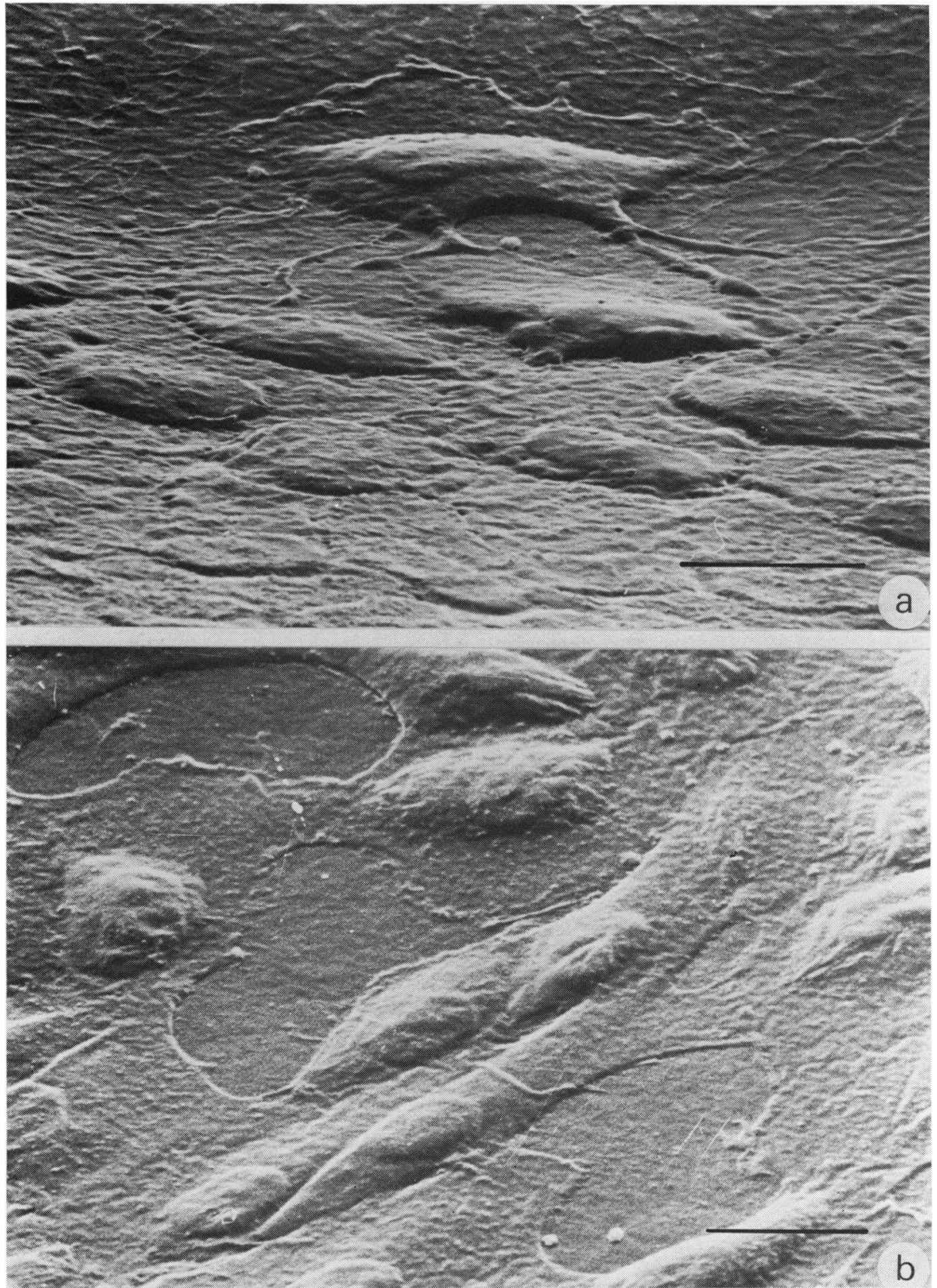

Fig. 4 Scanning electron micrographs of dedifferentiated endothelial cells associated with a large area of damage. (a) The edge of a large lesion 4 hours after exposure showing the dissociation of adjacent cells and the development of complex pseudopodia on all aspects of these migratory cells. (b) The centre of a large lesion 8 hours after exposure showing the presence of spindle-shaped fibroblast-like cells, and their organisation into a 'pig-skin graft'-like covering of Descemet's membrane. In this particular lesion 3 denuded areas of Descemet's membrane are apparent. 
cell debris within their path. Thus remnants of the basal membranes of ruptured cells became dissociated from Descemet's membrane. The tips of the pseudopodia often showed membrane specialisations such as macula adherens, and these were even formed in association with debris (Fig. 5a). Unlike the observations made on these processes in acute lesions, by 1 hour the cytoplasm in the region contained many organelles. As seen in SEM, cells participating in rosette formation became wedgeshaped, with their points towards the centres of the lesions. Between 1 and 4 hours after exposure and during this period of reorganisation the cytoplasm of these cells underwent significant changes, with a marked proliferation of ribosomal particles and an increase in the amount and distribution of smooth endoplasmic reticulum (Fig. $5 b$ ). This abnormal accumulation of organelles was more striking because of their correspondingly atypical distribution, with a zone-free of substructure adjacent to the apical membrane of the cells. The topography of the apical membrane itself was also atypical. Microvilli were absent in most of these cells, but the apical membrane was convoluted into large protrusions and contained many pinocytotic-like specialisations. These latter were formed by extremely complex cytoplasmic processes and were more common over the thicker portion of the wedge-shaped cells. In rosettes which had completely re-covered the lesion area intercellular contacts were redeveloped, and multiple desmosomal complexes between such newly juxtapositioned cells were a common finding. Conversely, focal membrane specialisations in association with Descemet's membrane were absent, and in many cases subcellular spaces were apparent. Neither of these membrane specialisations persisted in samples examined more than 8 hours after exposure, and within 24 hours the original sites of rosettes were extremely difficult to distinguish. At 48 hours after exposure such lesion sites were undetectable.

In large lesions similar changes were seen in both organelle numbers and distribution. However, in these lesions the migratory cells extended pseudopodia-like processes in all directions. Descemet's membrane was rarely damaged, but in a few lesions fibrin was deposited on the denuded surface. Debris was cleared from Descemet's membrane in a similar fashion to that of small lesions. In some lesions polymorphonuclear leucocytes were found in association with Descemet's membrane, and these generally had endothelial processes covering them (Fig. 5c). Within 48 hours most large lesions were re-covered with normal-looking endothelial cells and could be distinguished only by the atypical undulations of the surface membranes of these cells.
In some few instances endothelial cells were seen to contain lipid droplets and degenerative electrondense inclusions. Mitotic figures were not observed in any of the samples examined during the present study.

MEASUREMENTS OF CORNEAL THICKNESS Measurements of corneal thickness by specular microscopy showed that corneal swelling resulted from all ultrasonic exposures (Fig. 6). In vitro experiments showed that both the degree and rate of swelling were a function of the duration of the ultrasonic exposure. Thus 2 minutes of ultrasound resulted in a swelling of approximately $5 \mu \mathrm{m} / \mathrm{h}$, while a 5 - or 10-minute exposure increased this rate to $30 \mu \mathrm{m} / \mathrm{h}$. In the case of the 2-minute exposures to ultrasound there was some indication that the corneas had ceased to swell after the first $3 \frac{1}{2}$ hours (Fig. 6). This is about 1 hour after the time many of the lesions produced by such an injury would have been re-covered by endothelial rosette formation (Fig. 1e). After both 5- and 10-minute exposures to ultrasound no indication of a cessation or reduction in swelling rate was recorded during the 4-hour periods of observation.

In all our experiments there was a tendency for the corneas of control eyes to thin during observations under the specular microscope (Fig. 6). This change in thickness was never more than $50 \mu \mathrm{m}$ and and probably resulted from a temperature reversal effect. That is, during the transport period the corneas probably cooled and therefore absorbed water. Thus when they were placed on the heated chamber of the specular microscope, this accumulated water was pumped out.

Measurements on the corneas of the experimental animals in the in vivo series were carried out on enucleated eyes immediately after death, and all showed swelling during the first 8 hours after exposure. Within 24 hours of the ultrasonic injury experimental and control corneas were of equal thickness.

\section{Discussion}

These studies show that the integrity of the corneal endothelium is compromised by ultrasonic radiation with a beam geometry and irradiance identical to that commonly employed during phacoemulsification procedures. The induced lesions are sufficient in both number and distribution to disturb the equilibrium of the endothelial pump, which results in hydration of the stroma and corneal swelling. For any given irradiance the amount of induced damage is dependent on the duration of the ultrasonic exposure (Olson et al., 1978), and this 

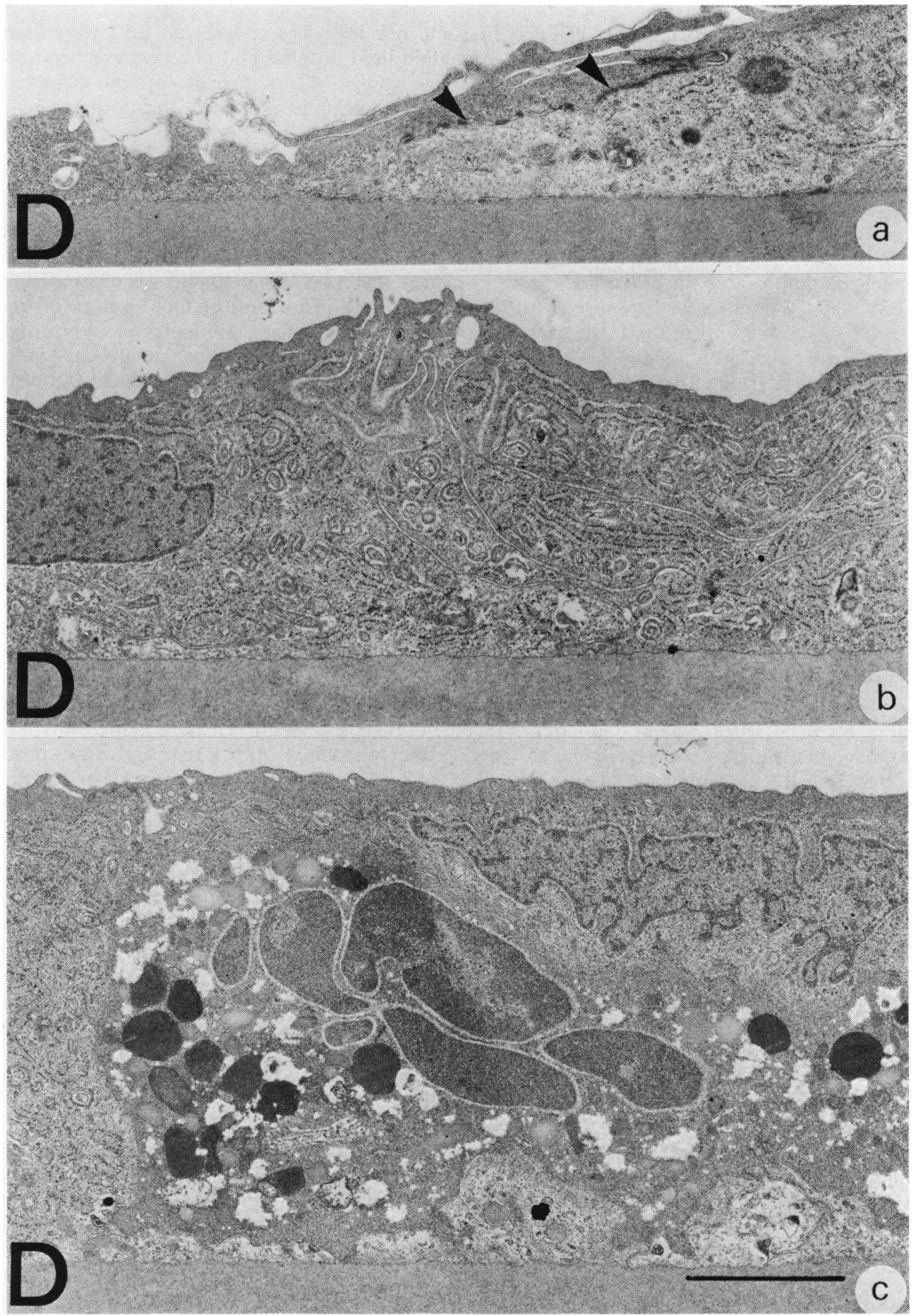

Fig. 5 (a) Transmission electron micrograph of the tip of a pseudopodia-like process from one of the endothelial cells participating in rosette formation. Membrane specialisations, macula adherens, are seen (arrowed) with cytoplasmic densifications in both the viable cell and in the debris of a ruptured cell. D is Descemet's membrane. (b) Transmision electron micrograph of an endothelial cell towards the centre of a rosette 2 hours after ultrasonic exposure. The cell is thinned, has an undulating surface membrane, and contains an extensively proliferated ribosomal system.

(c) Transmission electron micrograph of the edge of a large lesion 8 hours after ultrasonic exposure, showing the presence of a polymorphonuclear leucocyte situated on Descemet's membrane (D). The blood cell is covered by an endothelial cell. Bar marker $=2 \mu \mathrm{m}$ 


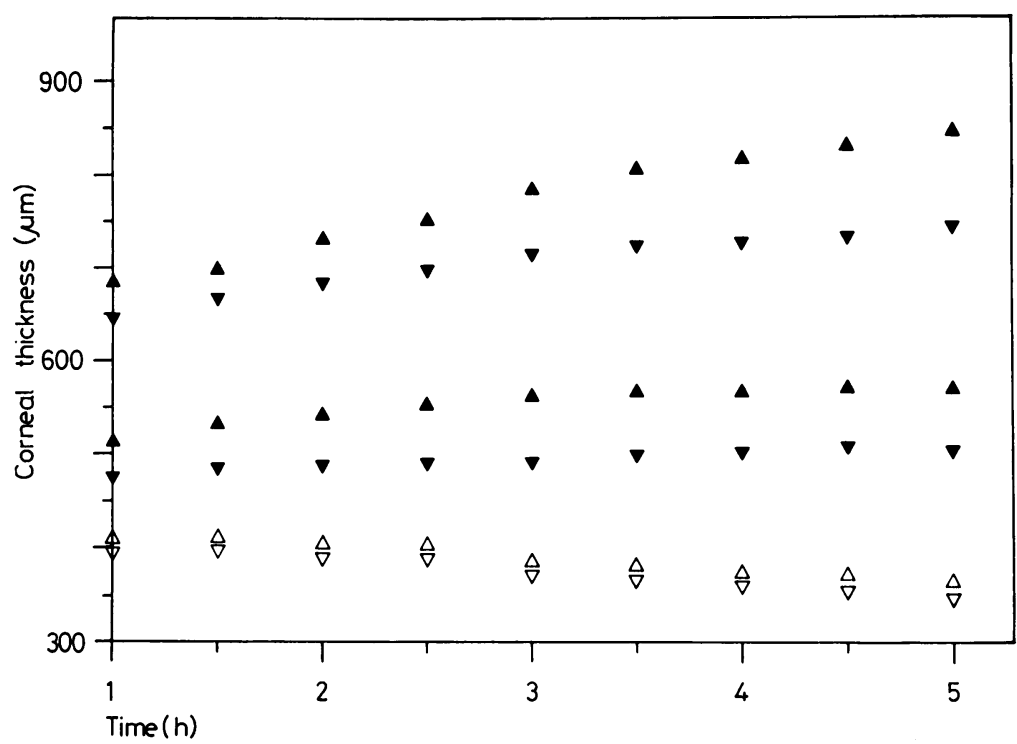

Fig. 6 Graph showing the variation of corneal thickness with time as measured on the specular microscope. The range of the control readings are indicated by open triangles. The upper series of closed triangles represents the range of swelling values obtained from corneas exposed to 10 minutes of ultrasound and the lower series of closed triangles the range of values obtained after a 2-minute exposure to ultrasound. The 10-minute exposures resulted in a strong continuous swelling during the period of observation, while the 2-minute exposures ceased to swell during the third hour after exposure finding is reflected in the rates of corneal swelling (Fig. 6). However, a maximum rate of swelling $(30 \mu \mathrm{m} / \mathrm{h})$ was observed after a 5-minute exposure of ultrasound, and this is in approximate agreement with figures determined after the complete phacoemulsification procedure carried out on rabbits (McCarey et al., 1976).

The endothelial response to ultrasonic injury and to a variety of different types of traumatic insult is to attempt to re-cover denuded areas of Descemet's membrane and thus re-establish the dynamic equilibrium of fluid transport. The repair processes are similar in different species, but the capacity and rates of such processes may differ significantly (van Horn et al., 1977). In the present study a 2-minute exposure of ultrasound resulted in a profusion of small lesions with a predominantly rosette-type repair process. The kinetic mechanisms and time course for the repair of these lesions were identical to those previously observed in the rabbit in response to either air in the anterior chamber (Leibowitz et al., 1974) or the administration of glycerin drops (Sherrard, 1976). This process seems to be confined to cells immediately adjacent to those that are compromised, and consists of a rapid growth or encroachment phase followed by a slower reorganisation period in which the cells return to the typical endothelial mosaic appearance. In our rabbits junctional complexes were observed between endothelial cells within an hour of membrane contact. It may, therefore, be of significance that the swelling rate of cornea exposed to 2 minutes of ultrasound reached a plateau at a similar period after the completion of rosette formation. The subsequent reorganisation of the endothelial mosaic must entail a degree of cell growth, and the process must be widespread, as it was not possible to identify enlarged cells in discrete areas within 48 hours or 1 week of exposure.

The capacity of the rabbit corneal endothelial cells for dedifferentiation and rapid growth was clearly demonstrated in the large lesions, where within 8 hours of insult fibroblast-like forms were seen migrating into the damaged area. As specular microscopy was carried out for only 5 hours after the injury, the dynamic effects of such reendothelisation on corneal thickness were not studied. The rate at which reparative processes began seems to be related to lesion size, as the initial changes associated with rosette formation occurred within 1 hour, those with large lesions took up to 8 hours, and lesions $6 \mathrm{~mm}$ in diameter caused by either alkali burns (Matsuda and Smelser, 1973) or cryocautery (Sanchez and Polack, 1974) took up to 48 hours before migratory endothelial cells became apparent. In our lesions denuded areas of Descemet's membrane were always completely re-covered within 24 hours; in the larger lesions reported in these earlier studies complete repopulation of the injured area took 5 to 7 days. In the present study, although the endothelial cells assumed a fibroblast-like appearance as they migrated into the damaged area, they did not lay down collagen fibrils, and no reduplication of Descemet's was observed. This would suggest that even gross ultrasonic injury within a 10-minute exposure period does not result in damage of the same magnitude as transcorneal freezing or alkali burns. This finding is further supported by 
the lack of mitotic figures in our study. It appears that the cell loss resulting from ultrasound represents a small fraction of the endothelial surface area and one that can be replaced by cell growth. We also confirm the observations of Matsuda and Smelser (1973) in that the fibroblast-like cells redifferentiate into endothelial cells once the damaged area is completely repopulated.

The origin of endothelial cells in wound repair has been the subject of intense discussion in the literature, with some writers implicating cell division at the wound edge and others suggesting central replacement by endothelial ingression resulting from mitotic activity at the limbus. Different species seem to have different capacities for endothelial replacement. In the present study all lesions have been repopulated by a process of cell growth. However, in more extreme lesions the rabbit endothelium seems to be able to initiate mitotic cell division around the edges of wounds (van Horn et al., 1977). In contrast in some other species such mechanisms of cell division seem poorly developed, and the repopulation is almost exclusively dependent on cell growth. This may explain why extensive corneal lesions in cat and monkey (Capella, 1972) take longer to heal than those of rabbit. It is also suggested that the reduction in endothelial cell numbers and their increase in size with increasing age in humans results from a similar lack of cell division in response to injury (Bourne and Kaufman, 1976; Laing et al., 1976). These findings have led van Horn et al. (1977) to conclude that cat is a more useful model in which to study induced endothelial changes. While agreeing with this conclusion, we feel that the rabbit is particularly useful in studying the effects of ultrasound, as its eye is more sensitive to this type of radiation than the human eye (Olson et al., 1978), and therefore an innate safety factor is present in any comparative studies.

In a clinical setting the first postoperative examination is usually made approximately 24 hours after surgery. At this time many of the smaller endothelial defects will have been repaired by rosette formation, and, while the repair of larger lesions will be slower, they are far fewer in number than in the rabbit. These findings may well explain the low incidence of residual corneal oedema resulting from the phacoemulsification of cataractous lens.

We would like to thank Professor Barrie R. Jones and Dr E. Sherrard for guidance and helpful advice during this project. We are grateful to $\mathrm{Mr} \mathrm{P}$. L. Ansell, Miss E. Clarke, and Mr J. Haddington for their technical assistance and to Mrs W. Pennel for secretarial help. The transmission electron micro- scope was provided by the Wellcome Trust, and some financial support was provided by the Pocklington Trust.

\section{References}

Binder, P. S., Sternberg, H., Wickham, M. G., and Worthen, D. M. (1976). Corneal endothelial damage associated with phacoemulsification. American Journal of Ophthalmology, 82, 48-54.

Bourne, W., and Kaufman, H. E. (1976). Endothelial damage associated with intraocular lenses. American Journal of Ophthalmology, 81, 482-485.

Capella, J. A. (1972). Regeneration of endothelium in diseased and injured corneas. American Journal of Ophthalmology, 74, 810-817.

Cleasby, G. W., Fung, W. E., and Webster, R. G. (1974). The lens fragmentation and aspiration procedure (phacoemulsification). American Journal of Ophthalmology, 77, 384-387.

Hiles, D. A., and Hurite, F. G. (1973). Results of the first year's experience with phacoemulsification. American Journal of Ophthalmology, 75, 473-477.

Hodson, S., and Miller, F. (1976). The bicarbonate ion pump in the endothelium which regulates the hydration of rabbit cornea. Journal of Physiology, 263, 563-576.

Kelman, C. D. (1974). Symposium: Phacoemulsification: summary of personal experience. Transactions of the American Academy of Ophthalmology and Otolaryngology, 78, 35-38.

Laing, R. A., Sandstrom, M. M., Berrospi, A. R., and Leibowitz, H. M. (1976). Changes in the endothelium as a function of age. Experimental Eye Research, 22, 587-594.

Leibowitz, H. M., Laing, R. A., and Sandstrom, M. M. (1974). Corneal endothelium: the effect of air in the anterior chamber. Archives of Ophthalmology, 82, 227-230.

Matsuda, H., and Smelser, G. K. (1973). Endothelial cells in alkali-burned corneas. Archives of Ophthalmology, 89, 402-409.

Maurice, D. M. (1968). Cellular membrane activity in the corneal endothelium of the intact eye. Experientia, 24, 1094-1095.

McCarey, B. E., Polack, F. M., and Marshall, W. (1976). The phacoemulsification procedure. I. The effect of intraocular irrigating solutions on the corneal endothelium. Investigative Ophthalmology, 15, 449-457.

Oh, J. O., and Evans, C. A. (1960). Suppressive effects of pyralamine maleate and d-lysergic acid diethylamide (LSD-25) on early corneal lesions produced in vitro by Newcastle disease virus (NDV) and compound 48/86. Virology, 10, 127-143.

Olson, L. E., Marshall, J., Rice, N. S. C., and Andrews, R. (1978). The effects of ultrasound on the corneal endothelium: I. The acute lesion. British Journal of Ophthalmology, 62, 134-144.

Polack, F. M., and Sugar, A. (1976). The phacoemulsification procedure. II. Corneal endothelium changes. Investigative Ophthalmology, 15, 458-469.

Sanchez, J., and Polack, F. M. (1974). Effect of topical steroids on the healing of corneal endothelium. Investigative Ophthalmology, 13, 17-22.

Sherrard, E. S. (1976). The corneal endothelium in vivo: its response to mild trauma. Experimental Eye Research, 22, 347-357.

van Horn, D. L., Sendele, D. D., Seideman, S., and Buco, P. J. (1977). Regenerative capacity of the corneal endothelium in rabbit and cat. Investigative Ophthalmology (Visual Sciences), 16, 597-613. 